\title{
Transfer of calibration in length perception by dynamic touch
}

\author{
ROB WITHAGEN \\ Vrije Universiteit, Amsterdam, The Netherlands \\ and \\ CLAIRE F. MICHAELS \\ Vrije Universiteit, Amsterdam, The Netherlands \\ and University of Connecticut, Storrs, Connecticut
}

\begin{abstract}
Earlier studies suggested that the calibration of actions is functionally, rather than anatomically, specific; thus, calibration of an action ought to transfer to actions that serve the same goal (Rieser, Pick, Ashmead, \& Garing, 1995). In the present study, we investigated whether the calibration of perception also follows a functional organization: If one means of detecting an information variable is recalibrated, are other means of detection recalibrated as well? In two experiments, visual feedback was used to recalibrate perceived length of a rod wielded by the right hand; the recalibration was found to transfer to length perception with the left hand. This implies that calibration in perception is organized functionally rather than anatomically, and supports the general view that calibration applies to functional systems.
\end{abstract}

Adaptive behavior requires that animals perceive and utilize the action possibilities of their environments. First, the animal must detect perceptual information that supports veridical perception or that can guide the intended action. The detection of a useful variable, however, is a necessary but not a sufficient condition for veridical perception or adaptive behavior. Useful variables can appropriately constrain the perception or action, but the perception or action should also be adequately scaled to the perceptual information. We use the term calibration to refer to the process that establishes and maintains the appropriate relation between the exploited information variable and the consequent perception or action (Jacobs \& Michaels, 2005).

Recently, calibration in perception and action has received considerable attention (see, e.g., Adolph \& Avolio, 2000; Bingham, Zaal, Robin, \& Shull, 2000; Jacobs \& Michaels, 2005; Mark, 1987; Riley \& Turvey, 2001; Wagman, Shockley, Riley, \& Turvey, 2001; Withagen \& Michaels, 2002). Although attempts have been made to develop a theory of calibration in perception-action (see,

We thank the University of Nijmegen for making the rods available to us, Idsart Kingma for the use of the response rail, and Hans de Koning for technical support. We thank Chris Pagano for providing us with the data from Experiment 1 of Pagano, Fitzpatrick, and Turvey (1993). We are grateful to Raoul Bongers, Len Mark, Anke van Mourik, Suzanne Nooy, Michael Riley, Frank Zaal, and three anonymous reviewers for helpful comments on an earlier draft, and to members of the Center for the Ecological Study of Perception and Action, University of Connecticut, for helpful suggestions and comments on the study. Correspondence concerning this article and reprint requests should be addressed to R. Withagen, who is now at the Center for Human Movement Sciences, University of Groningen, P. O. Box 196, 9700 AD Groningen, The Netherlands (e-mail: r.withagen@ppsw.rug.nl). e.g., Redding \& Wallace, 1997), thus far the question of the entity, process, or disposition to which calibration applies has received scant attention. It seems to us that establishing the object of calibration is an important step in developing a theory of calibration. A suggestion that is of interest in this regard is that calibration of actions is functionally organized (Rieser, Pick, Ashmead, \& Garing, 1995). This suggestion is based on the observation that a motor outcome can be realized by different actions involving different anatomical substrates, a phenomenon referred to as motor equivalence (see, e.g., Hebb, 1949; Lashley, 1930). As an example, to get to a place, a human being can walk, crawl, sidestep, and so on. The idea of the functional organization of calibration in action holds that the recalibration of one action generalizes to the other actions that achieve the same outcome, regardless of whether the actions are realized by the same effectors. Thus, for example, the functional model would predict transfer of calibration between walking, crawling, sidestepping, walking on hands, and other actions that serve the function of changing place in the environment. Rieser et al. (1995) contrasted this functional model with the limb-effector model, which holds that calibration applies to the effector by which the action is realized (see Anstis, 1995). Thus, for instance, in case of recalibration of walking, the calibration applies to the legs. Suggesting that calibration is specific to the anatomical structure performing the action (i.e., limbs), we will refer to the limbeffector model as the anatomical hypothesis. Anatomical specificity of calibration entails that calibration transfers to other actions that are performed with the same effector, regardless of the goals that the actions serve. Hence, the limb-effector model holds that calibration is indiffer- 
ent to functions and specific to limbs, whereas the functional model states that calibration is indifferent to limbs and specific to functions. Thus, the two models make different predictions about transfer of calibration.

To date, several transfer studies have provided evidence for a functional organization of calibration. Rieser et al. (1995) presented walkers with a discrepancy between their biomechanical walking speed and the optically specified speed at which they traveled through the environment. They found that after this "rearrangement phase," when asked to walk to a seen place without vision, participants walked too far or not far enough, depending on rearrangement type. This recalibration was shown not only by walking but also by sidestepping. The recalibration, however, was found not to transfer to throwing to a place, nor did the recalibration of throwing to a place transfer to walking. Although these results can be explained by both the anatomical and the functional hypotheses, Rieser et al. found evidence in favor of the latter in that the recalibration of walking to a seen place without vision did not transfer to turning in place, nor did the recalibration of turning in place transfer to walking. Berry and Rieser (1999) also rejected the limbeffector model in favor of the functional model by showing transfer of calibration between functionally equivalent actions performed with different limbs. They induced a recalibration of turning in place by presenting to participants a discrepancy between the optically specified turning rate and the biomechanically specified turning rate. They found that recalibration of turning in place with the legs partially transferred to turning in place with the arms, indicating that calibration is specific not to limbs but to the function of turning in place. Withagen and Michaels (2002) provided further evidence in favor of the functional hypothesis by showing that the recalibration of walking to a seen place without vision generalized to crawling to a seen place without vision. And Bruggeman, Pick, and Rieser (2001) showed that when participants sitting on a turning carousel were asked to throw beanbags underhand to the opposite side of the carousel, the directional recalibration was seen not only with underhand throwing but also with overhand throwing. Walking direction, however, was not recalibrated. Transfer of calibration to actions that have the same motor outcome is, however, not always found. For example, in a number of studies in which participants had to reach to an object while wearing prisms that displace the optic array, there was calibration of the exposed arm but not of the unexposed arm (see, e.g., Cohen, 1967; Harris, 1963; see also Anstis, 1995, for an illustration in locomotion).

Just as a motor outcome can be realized in several ways, it seems that animals are, in at least some cases, also capable of detecting a particular information variable by multiple means involving different anatomical structures (J. J. Gibson, 1966, 1979). That is, the function of detecting a particular information variable can also be realized in several ways. A simple illustration of this, and the one we exploit in the experiments to follow, is perception of length by dynamic touch. Human beings can perceive the length of a rod by holding it in the hand and wielding it (see, e.g., Solomon \& Turvey, 1988). The variable that informs this perception can, however, be detected in multiple ways involving different anatomical assemblages. Moreover, the haptic perceptual instrument, as it is termed, is described by Carello, Fitzpatrick, Domaniewicz, Chan, and Turvey (1992) as being softly assembled, which means that it is a functional organization assembled temporarily over possibly different anatomical structures. For instance, one can hold the rod in either the right or the left hand, and it can be wielded around the wrist, the elbow, the shoulder, or all these joints (see Pagano, Fitzpatrick, \& Turvey, 1993). Or one can hold the rod with one hand, two hands, or hand and knee (Carello et al., 1992). Thus, at the level of the detection of a particular information variable, there is reason to assume multiple realizability of function. ${ }^{1}$

Given that the function of detecting a particular information variable is multiply realizable, one can ask whether calibration in perception is functionally organized as well. Such a functional organization of calibration in perception would imply that the recalibration of the detection of information by one means transfers to the detection of the same information by other means. The hypothesis of the anatomical specificity of calibration, on the other hand, predicts no transfer of calibration from one anatomical structure by which the information can be detected to another. The present experiments were conducted to test these hypotheses.

As was noted above, the perceptual task we used to test for transfer of recalibration was the perception of rod length by dynamic touch. The participant is asked to hold one end of an unseen rod and is to report the rod's length. As has been shown repeatedly, individuals have a definite and reportable impression of length of a handheld, wielded rod (for overviews, see Turvey, 1996, and Turvey \& Carello, 1995). Research in dynamic touch has concerned itself primarily with revealing the informational variables that constrain perception. The measure of success in capturing an informational basis is whether or not the informational basis explains the variance in judgments, either within individuals or in averages over individuals. Thus, it is the fits of regression lines, rather than their coefficients, that are the most important data. The metrical accuracy of reports and, thus, the issue of calibration have usually not been a matter of concern. Nevertheless, dynamic touch is a convenient paradigm for studying perceptual calibration in general and transfer of perceptual calibration in particular. First, given the exploited information, different calibration is needed for rods of different densities; second, there exist convenient methods of testing for transfer of recalibration.

The fact that different calibration is needed for rods made of materials with different densities is demonstrated by the fact that rods of the same length but made of materials of different densities are perceived to be of different lengths (Fitzpatrick, Carello, \& Turvey, 1994; Solomon \& Turvey, 1988; Withagen \& Michaels, 2001). Thus, the values of the calibration constants that would 
yield metrically correct length perception differ for rods made of different materials.

Our point of departure is that the length perception depends on the inertia tensor (see, e.g., Fitzpatrick et al., 1994), so that calibration would be to the value of that tensor. Recently, however, there has been some debate about what variable governs length perception. Kingma, Beek, and van Dieën (2002) have suggested that length perception might be constrained instead by a combination of static moment and mass. It is important to note that for our purposes it does not matter whether the inertia tensor or static moment plus mass constrains length perception; what matters is that the value of the variable exploited differs for rods that are of equal length and different densities. Hence, by using a type of rod for which perceivers are not initially calibrated (i.e., their reports of its length are relatively, but not absolutely, correct), one can introduce feedback to study if and how length perception by dynamic touch can be recalibrated.

Second, the dynamic touch paradigm is convenient for the study of transfer of recalibration because there are easily separable anatomical structures by which haptic length information can be detected: the left hand versus the right hand. Thus, if calibration in perception is functionally organized rather than anatomically specific, then the recalibration of the detection of a particular information variable by one hand should transfer to the detection of the same information by the other hand.

\section{EXPERIMENT 1}

In Experiment 1, we had two goals. The first and primary goal was to test whether or not the recalibration of the right hand's length perception by dynamic touch transfers to the left hand's length perception by dynamic touch. We used a pretest-calibration-posttest design. In the test phases, the participants were asked to report the lengths of unseen, hand-held, wooden rods with both the right and the left hands. An earlier study in which these rods were used showed that their lengths are underestimated by most participants (Withagen \& Michaels, 2001). To induce the recalibration of length perception with the right hand, the participants were given visual feedback following their length estimations in the calibration phase. To determine whether calibration occurred and whether it transferred to the contralateral hand, we compared performance on the pretest with performance on the posttest for both the right and the left hands.

The second, and lesser, goal was to demonstrate that length as perceived by dynamic touch can be recalibrated and to establish that the calibration depends on feedback. To this end, a second group of participants received no feedback. The importance of feedback was assessed through a comparison of the two groups.

\section{Method}

Participants. Twenty participants ( 12 men and 8 women) volunteered to participate; each gave their informed consent. Their ages ranged from 21 to 34 years. All were right-handed.
Apparatus and Materials. The apparatus was similar to that used by Solomon and Turvey (1988). The participant was seated in a chair. On each side of the chair was an armrest that supported one of the participant's forearms. On a tabletop in front of the chair was a rail with a planar surface attached. The participant could move this surface toward or away from himself or herself by rotating a small wheel. Between each armrest and the rail was a curtain that prevented the participant from seeing the rod he or she wielded.

There were 10 wooden rods ranging in length from 10 to $100 \mathrm{~cm}$ in increments of $10 \mathrm{~cm}$. The rods were homogeneous and uniformly cylindrical with a diameter of $1.18 \mathrm{~cm}$. Each rod had an $11-\mathrm{cm}$ plastic handle affixed to it, which was separated from the rod by a disk. The masses of the rods were 14, 21, 27, 34, 40, 47, 53, 60, 67, and $73 \mathrm{~g}$.

Procedure. The experiment consisted of a pretest, a calibration phase, and a posttest. In each phase, the participants were to position the planar surface at the distance reachable with the hand-held rodthat is, in such a way that the rod's perceived distal end would coincide with the surface. The forearm was positioned so that the wrist extended just over the edge of the armrest. The forearm was held in this position by two vertical supports that were attached to the armrest just proximal to the wrist. The rod was to be held in such a way that the participant's thumb just touched the disk that separated the handle from the rod. The participants were allowed to wield the rod freely, with the exceptions that they were to maintain the forearm on the armrest and were not to touch the curtain with the rod. The position of the disk that separated the handle from the rod was taken as the reference point for our measurement of the perceived length. The perceived length was measured with a resolution of $0.5 \mathrm{~cm}$.

In both the pre- and posttests, perceivers made judgments on all 10 rods using each hand. Each rod was offered once to each hand; the order of presentation was randomized. The participants were not informed of the material of which the rods were made or the number of rods used. Blocks of five left-hand trials were alternated with blocks of five right-hand trials. Half of the participants started with right-hand trials, and the other half with left-hand trials. The participants were to position the planar surface at one of the ends of the rail after each trial.

The calibration phase consisted of six right-hand trials using different rod lengths in the following order: 30, 80, 20, 90, 10, and $100 \mathrm{~cm}$. After the participants positioned the planar surface at the felt distance reachable with the rod, the curtain was removed so they could see the rod. To induce the recalibration of perceived rod length, the participants were asked to wield the rod while looking at it. As in the test phases, the participants were to position the planar surface at one of the ends of the rail before each trial. In the control (no-feedback) group, the calibration phase was identical to that of the feedback group, except that no visual feedback was provided after the length judgment. Twelve participants were assigned to the feedback group and 8 to the control group.

Analyses. To examine the relationships between perceived length and actual length, we computed regression lines with perceived length as the dependent variable and actual length as the independent variable for each participant for each test phase and each hand. To test whether or not there were calibration effects, we performed analyses on the intercepts and slopes of the regression lines. ${ }^{2}$ The intercept is an indicator of the perceived position of the end of the rod when the actual length is zero and, thus, informs about the perceived hand position. For the length reports to be accurate, the intercept must be zero. Thus, when the intercept is not appropriately set in the pretest, a recalibration of the intercept is likely to occur. However, of more interest for our analyses are the slopes of the regression lines. The slope indicates how the perceived length is scaled to the actual length. Because of the nature of the variables that perceivers have been shown to exploit, the slope ought to be a function of the density of the rods. Rods made of a high-density material ought to yield a steeper slope than rods made of a lower density material. Given that the general underestimation of the length of the wooden rods is due mainly to a miscalibration of the 
slope, the main calibration effects are expected to be observed in the slopes.

\section{Results and Discussion}

The explained variances of the regression lines relating perceived length to actual length were quite high (mean $r^{2}=.91$ ), indicating that perceived length was a linear function of actual length. The slopes averaged across participants are depicted in Figure 1 together with error bars showing the standard deviations. Note that in the pretest the average slope is not appropriately calibrated for either hand. For the feedback group, the posttest average slope recalibrated considerably for both the right and the left hands. The average slopes even hint at a "super" transfer of calibration from the right to the left hand. The control group, in contrast, showed little change in average slope. We performed a repeated measures analysis of variance (ANOVA) on the slopes with test (pretest vs. posttest) and hand (right vs. left) as within-subjects factors and group (feedback vs. control) as a between-subjects factor. The main effect of hand $[F(1,18)=9.84, p<.01]$ indicates that the average right-hand slope was calibrated differently than the left-hand slope. The average slopes differ for the two groups $[F(1,18)=29.00, p<$ $.0001]$. The main effect of test $[F(1,18)=13.77, p<$ $.01]$ showed that perceived length was scaled to actual length differently in the pretest and in the posttest. The change in slope differed between the feedback and control groups, as is indicated by the test $\times$ group interaction $[F(1,18)=4.96, p<.05]$. The other interactions did not reach significance $(p s>.05)$. The test $\times$ group interaction indicates that the observed calibration effect is the result of the feedback. This is in line with earlier studies. In the absence of feedback, recalibration was found neither in the visual perception of distance (E. J. Gibson \& Bergman, 1954) nor in height and width perception by dynamic touch (Wagman et al., 2001). Those studies showed that only the variable error decreased in the absence of feedback.

The intercepts averaged across participants are depicted in Figure 2. A repeated measures ANOVA on the intercepts with test (pretest vs. posttest) and hand (right vs. left) as within-subjects factors and group (feedback vs. control) as a between-subjects factor revealed a significant effect of hand only $[F(1,18)=9.48, p<.01]$; the left hand was perceived to be posterior to the right hand. The absence of other significant effects $(p s>.10)$ suggests that perceived hand position did not significantly recalibrate and that there were no differences between the groups. Despite the fact that the intercept was not perfectly set in the pretest, the feedback provided in the calibration phase resulted in a recalibration only of slope, not of intercept.

Table 1 presents the coefficients of the regression lines for individual participants for each test phase and each hand. These scores provide a more detailed picture of the calibration effects and the relative absence of differences between the hands. Note that the length perception of Participant 11 did not improve with feedback. In contrast to the other participants of the feedback group, both the right-hand slope and the left-hand slope of this participant were less well calibrated in the posttest than in the pretest. Furthermore, note that the explained vari-

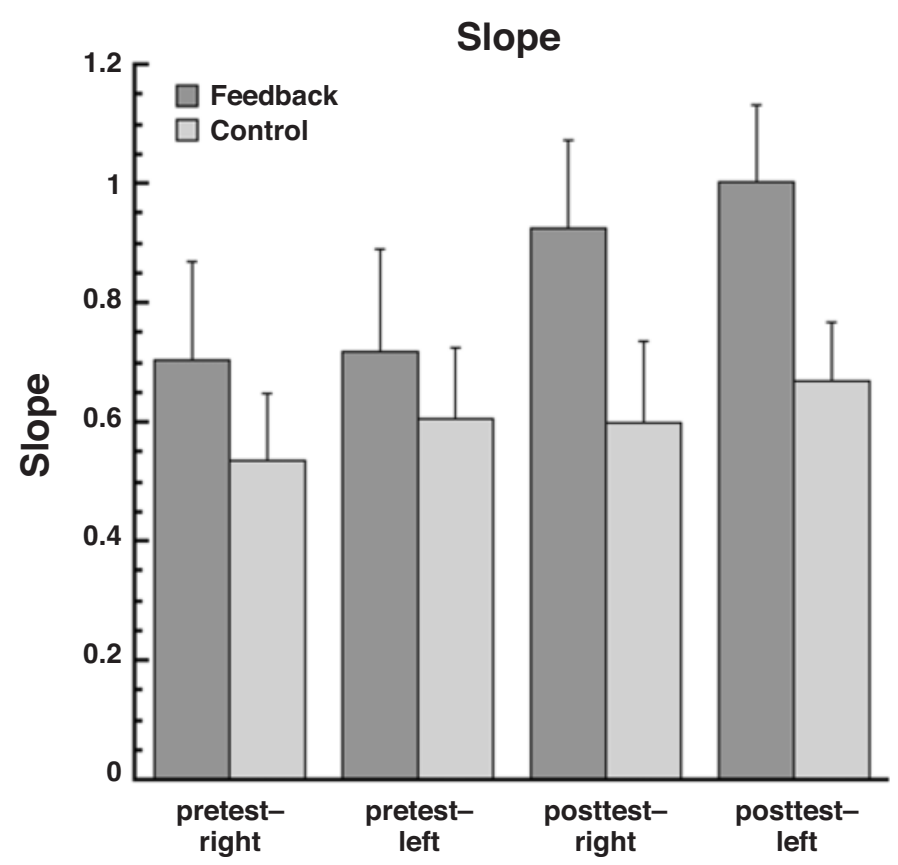

Figure 1. The averaged regression line slopes broken down by test phase, hand, and group in Experiment 1. The error bars indicate one standard deviation. 


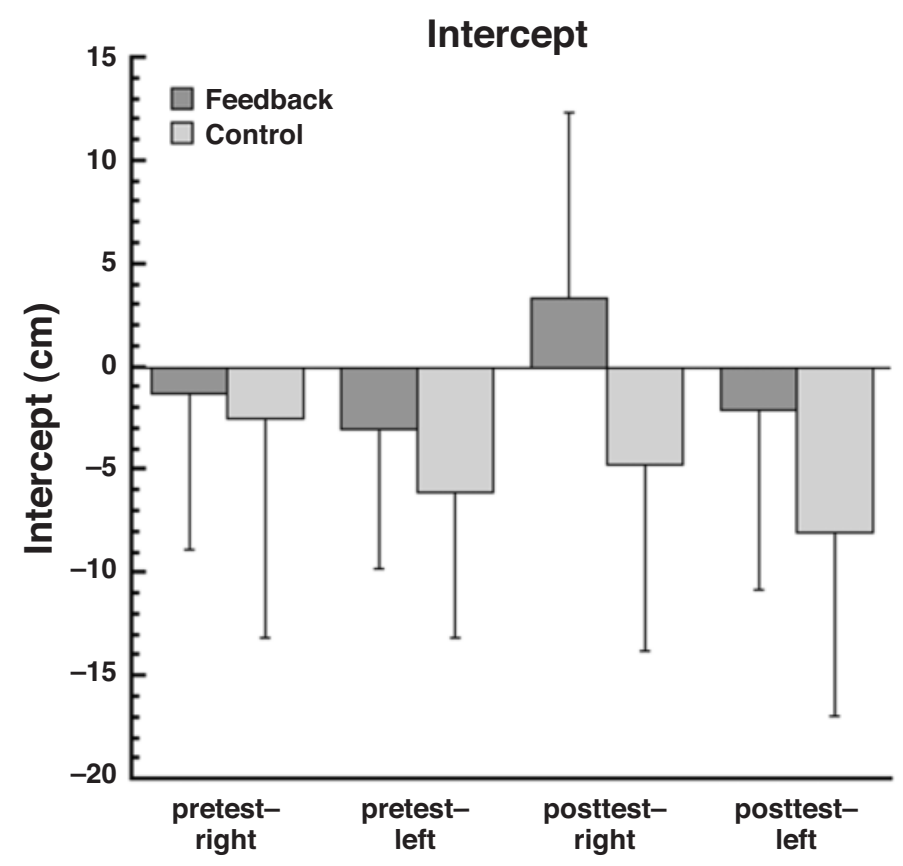

Figure 2. The averaged regression line intercepts broken down by test phase, hand, and group in Experiment 1. The error bars indicate one standard deviation.

ances of the regression lines were higher than .70, except for Participant 2 in the left-hand pretest $\left(r^{2}=.69\right)$ and Participant 7 in the right-hand pretest $\left(r^{2}=.54\right)$. Further analyses of these cells revealed that the two five-trial hand blocks lay on different straight lines (see Figure 3). The high degree of clustering around each line suggests that length perception with the particular hand was actually recalibrated during the pretest. Recall that in the test blocks, hand alternated in five-trial subblocks. It appears that wielding and making length judgments with the other hand - without feedback - can itself be a possible source of recalibration. The solid lines of Figure 3 capture

Table 1

Slopes, Intercepts, and Explained Variances of the Regression Lines of Perceived Length Against Actual Length in Experiment 1

\begin{tabular}{|c|c|c|c|c|c|c|c|c|c|c|c|c|c|}
\hline \multirow[b]{3}{*}{ Group } & \multirow[b]{3}{*}{ Participant } & \multicolumn{6}{|c|}{ Pretest } & \multicolumn{6}{|c|}{ Posttest } \\
\hline & & \multicolumn{3}{|c|}{ Right } & \multicolumn{3}{|c|}{ Left } & \multicolumn{3}{|c|}{ Right } & \multicolumn{3}{|c|}{ Left } \\
\hline & & $\mathrm{S}$ & I & $r^{2}$ & $\mathrm{~S}$ & I & $r^{2}$ & $\mathrm{~S}$ & I & $r^{2}$ & $\mathrm{~S}$ & I & $r^{2}$ \\
\hline \multirow[t]{12}{*}{ Feedback } & 1 & 0.70 & -1.2 & .95 & 0.70 & -7.9 & .95 & 0.82 & 11.7 & .97 & 1.00 & -3.1 & .96 \\
\hline & 2 & 0.83 & 7.3 & .96 & 0.64 & 1.0 & .69 & 1.10 & 0.2 & .93 & 1.20 & -14.4 & .96 \\
\hline & 3 & 0.87 & -3.7 & .95 & 0.86 & -7.2 & .95 & 0.91 & 11.1 & .93 & 0.83 & 10.7 & .91 \\
\hline & 4 & 0.62 & -6.0 & .90 & 0.54 & -5.9 & .81 & 0.83 & -3.8 & .93 & 0.87 & -7.4 & .91 \\
\hline & 5 & 0.63 & -5.1 & .82 & 0.60 & -1.5 & .91 & 1.10 & 6.9 & .85 & 1.00 & 7.4 & .87 \\
\hline & 6 & 0.61 & 1.6 & .90 & 0.79 & -7.8 & .98 & 0.81 & 10.4 & .95 & 0.96 & -0.7 & .98 \\
\hline & 7 & 0.59 & 3.4 & .54 & 0.70 & 3.1 & .87 & 0.81 & 15.2 & .90 & 0.96 & 6.5 & .88 \\
\hline & 8 & 1.10 & -15.7 & .96 & 1.00 & -10.3 & .82 & 1.00 & 5.1 & .94 & 1.10 & 7.4 & .85 \\
\hline & 9 & 0.55 & 6.8 & .78 & 0.52 & 7.8 & .89 & 0.96 & -3.8 & .93 & 0.86 & -6.6 & .94 \\
\hline & 10 & 0.52 & 11.0 & .95 & 0.55 & 9.1 & .95 & 1.08 & -10.6 & .94 & 1.19 & -12.1 & .97 \\
\hline & 11 & 0.87 & -9.3 & .77 & 1.03 & -7.2 & .88 & 0.62 & 9.3 & .88 & 0.88 & 0.0 & .89 \\
\hline & 12 & 0.65 & -3.9 & .96 & 0.73 & -9.4 & .92 & 1.1 & -11.2 & .91 & 1.10 & -12.6 & .94 \\
\hline \multirow[t]{8}{*}{ Control } & 13 & 0.65 & 0.5 & .94 & 0.76 & -6.8 & .98 & 0.65 & -2.0 & .99 & 0.66 & 3.4 & .98 \\
\hline & 14 & 0.73 & -16.2 & .94 & 0.78 & -16.6 & .98 & 0.71 & -18.1 & .93 & 0.76 & -18.2 & .94 \\
\hline & 15 & 0.51 & -2.0 & .88 & 0.51 & -1.4 & .72 & 0.75 & 0.5 & .96 & 0.69 & 0.9 & .84 \\
\hline & 16 & 0.49 & -15.8 & .95 & 0.49 & -7.4 & .88 & 0.40 & -10.5 & .80 & 0.47 & -11.1 & .93 \\
\hline & 17 & 0.37 & 16.5 & .81 & 0.47 & 4.7 & .84 & 0.40 & 11.0 & .95 & 0.64 & -2.6 & .93 \\
\hline & 18 & 0.49 & -5.5 & .92 & 0.65 & -13.1 & .87 & 0.60 & -12.6 & .94 & 0.66 & -20.3 & .85 \\
\hline & 19 & 0.60 & -2.3 & .92 & 0.57 & -8.3 & .84 & 0.70 & -5.0 & .91 & 0.81 & -13.1 & .97 \\
\hline & 20 & 0.46 & 4.5 & .99 & 0.64 & 0.2 & .97 & 0.59 & -1.4 & .96 & 0.65 & -3.2 & .96 \\
\hline
\end{tabular}



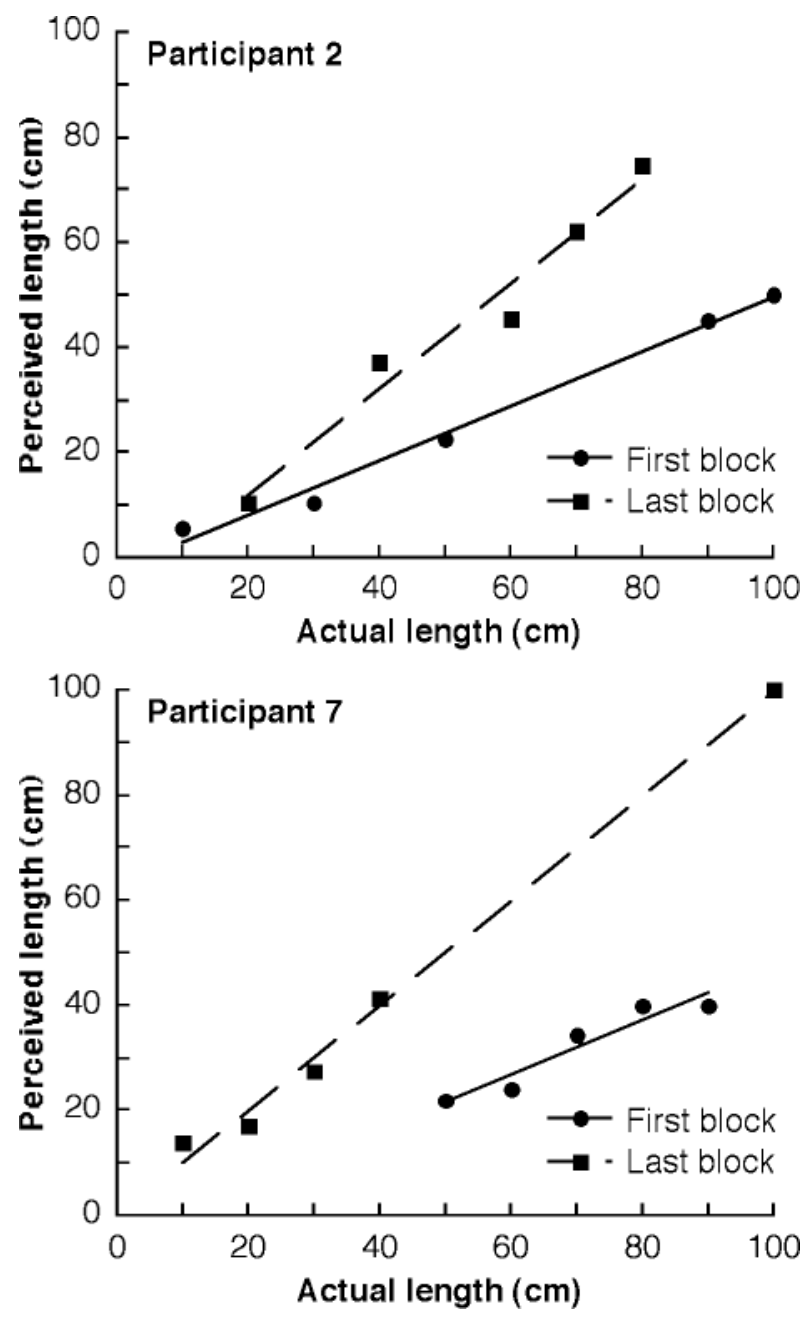

Figure 3. Perceived length as a function of actual length for Participant 2 in the left-hand pretest and Participant 7 in the right-hand pretest of Experiment 1. The circles represent the first five trials, and the squares represent the last five trials.

calibration before exposure of the contralateral hand. The contralateral hand, in turn, yielded a steeper slope, which seems to have increased the steepness of the calibration function of the first hand. Thus, the length perception with one hand might function as a possible source of "feedback" for the other hand, at least in some cases.

Note that for the control group, Table 1 shows that there were some small changes in slope within participants. However, there was no reliable trend across participants.

In sum, the main conclusions of Experiment 1 are that length perception via dynamic touch can be appropriately recalibrated by visual feedback and that that recalibration transfers to the contralateral hand.

\section{EXPERIMENT 2}

Experiment 1 showed that a restricted amount of feedback sufficed to recalibrate the perception of the lengths of wooden rods by dynamic touch so that the reports of length were correctly scaled. Furthermore, a complete transfer of calibration to length perception with the left hand was observed. However, given that the average slope in the pretest was 0.64 , not much recalibration was needed to correctly scale perceived length to actual length. Experiment 2 was conducted to test whether or not calibration also transfers if a more dramatic calibration was induced. A pilot study showed that the perception of the lengths of wooden rods with the right hand can be recalibrated to a scale of 1.5 times their actual lengths. ${ }^{3}$ In Experiment 2, we tested whether there is also a transfer of calibration from the right to the left hand when the righthand perception was to be calibrated to such a scale.

\section{Method}

Participants, Apparatus, and Materials. Nine participants ( 5 men and 4 women) volunteered to participate and gave their informed consent. Their ages ranged from 19 to 27 years. All were righthanded. None of the volunteers had participated in Experiment 1. The apparatus and rods were the same as those of Experiment 1.

Procedure. As in Experiment 1, we used a pretest-calibrationposttest design. The test phases were identical to those of Experiment 1 . The calibration phase was the same as in Experiment 1, except that after the participants made their judgments the feedback was given by positioning the planar surface at 1.5 times the actual rod length. Thus, in contrast to the calibration phase in Experiment 1 , the participants could not see the rod. To induce the recalibration, the participants were asked to wield the rod while looking at the planar surface. In contrast to Experiment 1, there was no control group (which would have been identical to the control group in Experiment 1).

\section{Results and Discussion}

As in Experiment 1, we computed the regression lines between perceived length and actual length for each participant for each test phase and each hand. The perceived length was a linear function of actual length, as is indicated by the high explained variances of the linear regression lines (mean $\left.r^{2}=.91\right)$. Participant 6 showed no recalibration of the length perception with the right hand (the slope was 0.67 in the pretest and 0.75 in the posttest, and the intercept was $-14.0 \mathrm{~cm}$ in the pretest and $-14.8 \mathrm{~cm}$ in the posttest), rendering it impossible to test whether there was transfer of calibration from the right hand to the left. Hence, we excluded this participant from tests of transfer. However, as is reported below, the qualitative pattern of results is not changed by inclusion of this participant.

The slopes averaged across participants are depicted in Figure 4. To test for transfer, we performed a repeated measures ANOVA on the slopes with test (pretest vs. posttest) and hand (right vs. left) as factors. There was a main effect of test $[F(1,7)=173.92, p<.0001]$, indicating that slope recalibrated. The average slope in the posttest approximated 1.5 , showing the ability of dynamic touch to recalibrate. There was no difference in slope between the hands $[F(1,7)=0.16, p>.1]$. There was no condition $\times$ hand interaction $[F(1,7)=0.38, p>.1]$, which implies that there was a complete transfer of recalibration of slope from the right to the left hand.

The intercepts averaged across participants are depicted in Figure 5. A repeated measures ANOVA on the 


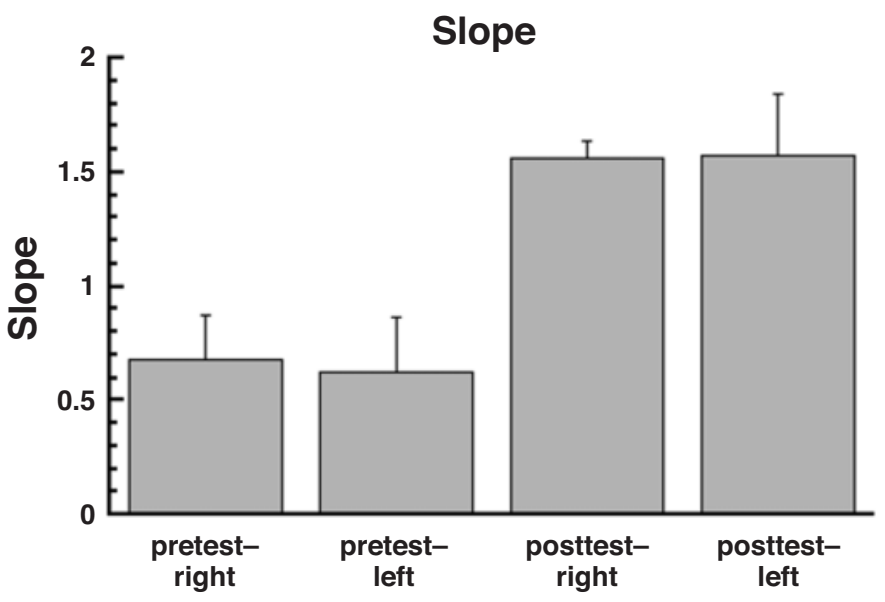

Figure 4. The regression line slopes averaged across participants for each test phase and each hand in Experiment 2. The error bars indicate one standard deviation.

intercepts with test (pretest vs. posttest) and hand (right vs. left) as factors revealed no significant effects ( $p \mathrm{~s}>$ .05 ), indicating that perceived hand position was not recalibrated and that there was no difference between hands. Thus, as in Experiment 1, the feedback resulted in a change only in slope, not in intercept. ${ }^{4}$

The regression coefficients for individual participants are provided in Table 2 . These scores illustrate the individual differences in calibration effects as well as the relative absence of differences between the hands. Note the calibration coefficients shown for Participant 3. The results of this participant illustrate just how miscalibrated perceived length by dynamic touch can be to begin with (in the pretest, the slope was 0.29 for the right hand and 0.24 for the left hand, indicating a calibration to rods made of dense material); nevertheless, feedback was sufficient for the system to recalibrate (in the posttest, the slope was 1.55 for the right hand and 1.13 for the left hand).

In sum, we concluded that if length perception by dynamic touch is recalibrated for the right hand, length perception by the left hand is also recalibrated, and to approximately the same extent.

\section{GENERAL DISCUSSION}

The experiments reported here were conducted to test whether there is a functional organization of calibration in perception - that is, whether the recalibration of the detection of an information variable by one anatomical structure generalizes to the detection of the information by other anatomical structures. The functional hypothesis was contrasted with the anatomical hypothesis, which

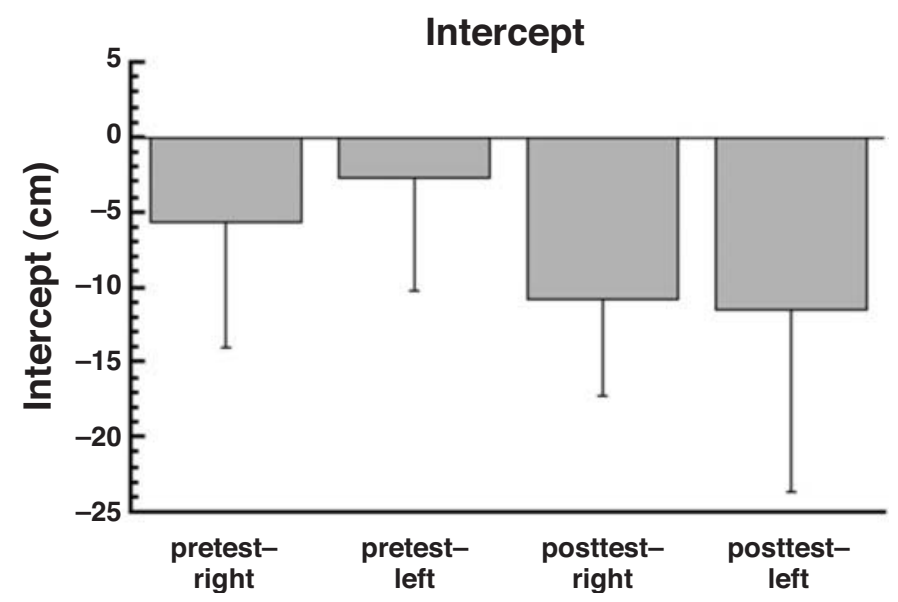

Figure 5. The regression line intercepts averaged across participants for each test phase and each hand in Experiment 2. The error bars indicate one standard deviation. 
Table 2

Slopes, Intercepts, and Explained Variances of the Regression Lines in Experiment 2

\begin{tabular}{|c|c|c|c|c|c|c|c|c|c|c|c|c|}
\hline \multirow[b]{3}{*}{ Participant } & \multicolumn{6}{|c|}{ Pretest } & \multicolumn{6}{|c|}{ Posttest } \\
\hline & \multicolumn{3}{|c|}{ Right } & \multicolumn{3}{|c|}{ Left } & \multicolumn{3}{|c|}{ Right } & \multicolumn{3}{|c|}{ Left } \\
\hline & S & I & $r^{2}$ & $\mathrm{~S}$ & I & $r^{2}$ & S & I & $r^{2}$ & S & I & $r^{2}$ \\
\hline 1 & 0.59 & 1.7 & .83 & 0.48 & 6.6 & .76 & 1.61 & -16.7 & .93 & 1.80 & -24.4 & .89 \\
\hline 2 & 0.70 & -5.5 & .98 & 0.56 & -1.1 & .92 & 1.50 & -9.2 & .92 & 1.51 & -16.3 & .97 \\
\hline 3 & 0.29 & -7.7 & .93 & 0.24 & -1.9 & .79 & 1.55 & -0.9 & .95 & 1.13 & 4.9 & .89 \\
\hline 4 & 0.78 & 2.1 & .92 & 0.93 & -3.0 & .91 & 1.56 & -3.4 & .84 & 1.89 & -10.5 & .92 \\
\hline 5 & 0.72 & -14.7 & .92 & 0.49 & -4.3 & .84 & 1.44 & -17.1 & .90 & 1.68 & -29.0 & .88 \\
\hline 6 & 0.67 & -14.0 & .89 & 0.82 & -20.0 & .94 & 0.75 & -14.8 & .91 & 0.87 & -16.3 & .95 \\
\hline 7 & 0.82 & -6.1 & .96 & 0.81 & -3.1 & .90 & 1.60 & -15.6 & .97 & 1.25 & -1.3 & .96 \\
\hline 8 & 0.94 & -19.0 & .93 & 0.93 & -18.9 & .93 & 1.66 & -15.8 & .93 & 1.61 & -15.4 & .94 \\
\hline 9 & 0.58 & 4.8 & .96 & 0.55 & 4.9 & .95 & 1.61 & -7.1 & .96 & 1.75 & 0.8 & .92 \\
\hline
\end{tabular}

Note-S, slope; I, intercept.

holds that recalibration is specific to the anatomical structure (i.e., limb) by which the function is performed. Thus, the anatomical hypothesis predicts no transfer to other anatomical structures or assemblages thereof. Our functional hypothesis regarding perception was derived from evidence that the calibration of actions is functionally organized - that is, that calibration of an action generalizes to actions that serve the same goal (see, e.g., Berry \& Rieser, 1999; Bruggeman et al., 2001; Rieser et al., 1995; Withagen \& Michaels, 2002). The paradigm we chose to test for transfer of calibration in perception was length perception by dynamic touch. This choice was motivated, first, by the fact that the hypothesized informational bases for length perception via dynamic touch require different calibration for rods made of materials with different densities and, second, by the fact that there are easily separable means - the two handsby which the information can be detected.

We performed two experiments, each with a pretestcalibration-posttest design. In Experiment 1, we found that length perception by dynamic touch could be appropriately rescaled to actual length through visual feedback. Transfer of calibration of length perception with the right hand to the left hand was observed. Experiment 2 showed that there was also transfer of calibration if the right-hand length perception of wooden rods was calibrated to a scale of 1.5 their length. We found the average posttest slopes of both the right and the left hands to approximate 1.5 , indicating transfer of calibration. We concluded that calibration is specific to the function of detecting length information yielding the percept, rather than to the hand that detects the information.

In what follows, we consider the relation between calibration and perceptual learning and how calibration of perception might be formalized. We conclude with speculations on what is being calibrated and what information might be involved.

\section{Calibration or the Education of Attention?}

Are our results instances of calibration, or are they better thought of as perceptual learning? Obviously, the answer rests in part on how perceptual learning and calibration are distinguished. The distinction we have adopted is that perceptual learning entails the education of attentiona change in the variable that informs the judgment (see, e.g., J. J. Gibson, 1966) - whereas calibration does not. We have presented our results as bearing on recalibration - the adoption of a new scaling relation between detected information and the perception (or, by extension, the action) that it informs. But do the reported results also yield to an explanation in terms of perceptual learning? We think not. Although a change in the variable that is exploited can yield a change in the slope and/or intercept of the regression line relating perceived length to actual length, the detection of another variable in and of itself does not result in appropriately scaled length perception. Recall that we used homogeneous and uniformly cylindrical wooden rods of different lengths. For a collection of rods so constrained, many variables specify length. For metrically correct length perception, participants must detect one of these specifying variables and calibrate so that perceived length is appropriately scaled to actual length. The high explained variances of the regression lines indicate that in both the pre- and posttests the participants detected one of the specifying variables (e.g., inertia tensor or static moment plus mass). It is, of course possible that the participants changed in the exploited specifying variable. However, even if the participants did so, recalibration is still required. Thus, the question is not whether calibration or the education of attention occurred, but whether calibration and the education of attention occurred. We find it more parsimonious to ascribe the changes only to the adjustment of the relationship between the perceptual information exploited and perceived length - that is, to recalibration.

\section{A Change in Perception or Judgment?}

We have described our results in terms of how perception changes as a function of feedback. But is there another possibility? As Heft (1993) argued, perceptionaction tasks can be performed with an "analytic attitude," with which one makes an analytic, reflective judgment. In the experiment reported here, such an analytic attitude would mean that the participants accurately reported not their perception but a reflective judgment based on it. That is, it might be possible that the length perception by 
dynamic touch was not really recalibrated but that the participants were merely assigning different response panel positions to the same perceived length. It is difficult to distinguish between these possibilities, and volunteered verbal reports about how the participants performed the task do not provide the required information. Because the participants did not know that the same rods were offered in the posttest and the pretest, nor when a particular rod was held, they could not answer the question of whether their length perception had changed. Moreover, participants in experiments are often unreliable in explaining how they perform tasks. This also was explicitly the case in a pilot study in which 1 participant volunteered that his perception did not change but that he always added about $15 \mathrm{~cm}$ in the posttest because of the underestimation revealed through feedback. However, examination of his performance in the posttest relative to the pretest showed that his slope increased by 0.82 and his intercept decreased by $13.2 \mathrm{~cm}$. Hence, either his verbal report about his strategy or that about his perception was wrong.

We tentatively conclude, therefore, that perception by dynamic touch, rather than mere judgment, was recalibrated. There are also logical arguments for this conclusion. After all, recalibration must be possible in the case of dynamic touch; otherwise, people would be calibrated for a particular kind of rod for their whole lives. Second, the perception of rod length by dynamic touch is likely to be recalibrated through visual information about this property. Studies using prismatic displacement provide ample evidence that perception can be recalibrated through visual feedback (see, e.g., Cohen, 1967; Harris, 1963, 1965; Riley \& Turvey, 2001), and there is no reason to suppose that this cannot occur in length perception by dynamic touch.

\section{Are the Hands Special?}

One might wonder whether the transfer of calibration between the left and right hands is special, given cortical interconnectivity between the brain parts that support the two hands. Would transfer occur to other joints around which a rod is wielded, for example? The data of Pagano et al. (1993) permit us to make an informal test of this. Pagano et al. compared the perceived lengths of rods wielded only around the wrist, only around the elbow, and only around the shoulder. A weak prediction of the functional hypothesis is that slopes of the regression lines for the three joints should be similar (as the slopes of the regression lines for the left and right hands of each individual tended to be similar in our pretests). Pagano et al. provided us with their raw data, and we computed the slopes, which are presented in Table 3. It is clear that there is less variation in slope within perceivers than there is between perceivers. We interpret this as at least a tentative demonstration that the left and right hands are not unique beneficiaries of transfer.

Given the cross joint results, we think one should not seek an explanation of the interhand transfer in handspecific mechanisms. If one were to push for a neural
Table 3

Slopes of Regression Lines Relating Perceived to Actual Length for Homogeneous Rods Wielded About the Wrist, Elbow, and Shoulder

\begin{tabular}{cccc}
\hline Participant & Wrist & Elbow & Shoulder \\
\hline 1 & 1.5 & 1.4 & 1.2 \\
3 & 0.8 & 0.7 & 0.8 \\
4 & 1.9 & 1.9 & 2.0 \\
5 & 0.5 & 0.7 & 0.7 \\
6 & 1.1 & 1.3 & 1.5 \\
7 & 1.3 & 1.1 & 1.1 \\
8 & 1.1 & 1.3 & 1.2 \\
\hline
\end{tabular}

Note-The slopes for Participant 2 were omitted because the regression line for the wrist accounted for less than $2 \%$ of the variance. All the reported slopes were from regression lines that accounted for at least $62 \%$ of the variance. The data were collected by Pagano et al. (1993).

correlate of transfer, the functional organization of calibration seems to point to a functional architecture of the brain. It seems reasonable to suppose that the functional organization of calibration is embodied by a change in brain areas that are specific to functions as opposed to anatomical structures such as, for instance, limbs. Recently, Gallese (2003) and colleagues have found evidence of the existence of such brain parts. In the macaque monkey's brain, certain cells are active any time the monkey successfully grasps an object, regardless of whether it grasps it with the hands, the mouth, or both. Hence, as Gallese asserted, these brain areas seem to be specific to goals or functions.

\section{What Is Calibrated?}

As we argued in the introduction, identifying the entity, process, or disposition to which calibration applies is an important step in a theory of calibration in perception and action. What does the fact that the recalibration of length perception with one hand transfers to this perception with the other hand mean in this regard? In the remainder of this article, we consider the implications of our findings for the suggestion that functional systems are recalibrated (see Withagen \& Michaels, 2002). The concept of functional system was introduced by Anokhin (1935) and developed by J. J. Gibson, (1966, 1979), Luria (1973), and Reed (1982, 1996) among others. Functional systems are to be defined in terms of function; they should not be thought of as a particular anatomical structure that executes a certain function. Instead, functional systems can, in general, manifest themselves in several ways involving different anatomical structures. Luria gave as an example the respiratory system:

[I]f the principal group of muscles working during respiration (the diaphragm) ceases to act, the intercostal muscles are brought up into play, but if for some reason or other they are impaired, the muscles of the larynx are mobilized and the animal or person begins to swallow air, which thus reaches the alveoli of the lung by a completely different route. (p. 28)

Hence, the function and the final result stay the same, but the way the function is performed and, thus, the 
anatomical structures involved may vary. Thus, functional systems can manifest themselves through different assemblages of anatomical structures (see, e.g., Bingham, 1988; Kugler, Kelso, \& Turvey, 1980; Kugler \& Turvey, 1987; Luria, 1973).

Although some progress has been made in the development of the concept of functional system (see, e.g., Reed, 1982, 1996), there is to date no accepted principle by which one can enumerate functional systems. The lack of a taxonomy of functional systems means we cannot prove that calibration applies to functional systems (see Withagen \& Michaels, 2002). However, calibration appears to apply to distinguishable ways of executing a particular function. In other words, transfer studies suggest the existence of different functional systems, each of which can be recalibrated independently. The present experiments, for instance, suggest that there exists a functional system that detects length information via dynamic touch and that this system can be recalibrated. Further tests of this conceptualization are needed, however.

First, for calibration to apply to the functional system that detects length information by dynamic touch, transfer between all the different means by which this information can be detected should occur. In the present experiments, we tested only two such means. Furthermore, they both were of the type that involve holding an end of the rod in the hand and wielding it about the wrist. A first, obvious test would be a formal test of transfer between joints in the Pagano et al. (1993) paradigm. Second, if calibration applies to the system responsible for perceiving length by dynamic touch, there should be no transfer of calibration to the perception of other environmental properties by dynamic touch. One might think, for instance, of the perception of the rod's heaviness, width, or center of percussion.

\section{The Information for Recalibration}

As was noted earlier, the calibration of an action has not always been shown to transfer to actions that serve the same goal (see, e.g., Cohen, 1967; Harris, 1963). Hence, the conclusion that calibration always applies throughout a functional system is too simple and must depend on other conditions.

A discovery interesting in this regard comes from prism studies: It has been shown that partial transfer from the exposed to the unexposed arm is found only under certain conditions (see, e.g., Cohen, 1967; Hamilton, 1964; Redding \& Wallace, 1988; Wallace \& Redding, 1979). Several investigations (see, e.g., Hamilton, 1964; Redding \& Wallace, 1988), for instance, found no transfer of calibration to the unexposed arm when head and trunk movements were restricted in the prism-wearing session. However, when the participant's head and trunk were free to move, transfer of calibration to the unexposed arm did occur, at least partially. The fact that transfer of calibration does occur under certain conditions might be interpreted to mean that particular information may be required for a functional system to calibrate.
There is a parallel to be drawn here with J. J. Gibson's $(1966,1979)$ theory of perception, which holds that the perception of an environmental property is the result of the detection of information that specifies that property. When such information is not available, perception will not and cannot occur. The same principle might hold for the calibration of a functional system: The recalibration of a functional system requires certain information, and when this information is not available, the functional system will not and cannot recalibrate. The nature of this information remains, however, to be discovered. Nevertheless, we might crudely sketch out some necessary characteristics of information for calibration, which may help guide the search. First, the information for calibration surely must bear on the relation between the perceptual judgment (or outcome of the action) and its feedback or environmental consequences. Second, it is surely a pattern over successive trials, at least in a task such as ours, in which discrete judgments are solicited. The linear information-perception relation observed here, for example, has two degrees of freedom - a slope and an intercept - and, therefore, a perceiver could not adequately recalibrate both on the basis of feedback about a single judgment. Third, the information that guides calibration must do just that: It cannot simply inform the perceiver of a mismatch but must specify the nature of the needed change. Finally, the aforementioned prism studies suggest that calibration information is more effective when it is obtained by, rather than imposed on, the perceiver, as J. J. Gibson (1966) suggested for perceptual information.

\section{REFERENCES}

Adolph, K. E., \& Avolio, A. M. (2000). Walking infants adapt locomotion to changing body dimensions. Journal of Experimental Psychology: Human Perception \& Performance, 26, 1148-1166.

ANOKHIN, P. K. (1935). Problema tsentra i periferii v sovremennoi fiziologii nervnoi deyatel'nosti [The problem of center and periphery in the contemporary physiology of nervous activity]. In P. K. Anokhin (Ed.), Problema tsentra i periferii v sovremennoi fiziologii nervnoi deyatel'nosti (pp. 1-70). Gorki, Russia: Gosizdat.

Anstis, S. (1995). Aftereffects from jogging. Experimental Brain Research, 103, 476-478.

BERRY, D. M., \& RIESER, J. J. (1999, August). Changes in rotational locomotion transfer from foot to hand. Paper presented at the Tenth International Conference on Perception and Action, Edinburgh.

Bingham, G. P. (1988). Task-specific devices and the perceptual bottleneck. Human Movement Science, 7, 225-264.

Bingham, G. P., ZaAl, F., Robin, D., \& Shull, J. A. (2000). Distortions in definite distance and shape perception as measured by reaching without and with haptic feedback. Journal of Experimental Psychology: Human Perception \& Performance, 26, 1436-1460.

Bruggeman, H., Pick, H. L., JR., \& Rieser, J. J. (2001, June). Directional recalibration in throwing. Poster presented at the Eleventh International Conference on Perception and Action, Storrs, CT.

Carello, C., Fitzpatrick, P., Domaniewicz, I., Chan, T.-C., \& TurVEY, M. T. (1992). Effortful touch with minimal movement. Journal of Experimental Psychology: Human Perception \& Performance, $\mathbf{1 8}$ 290-302.

CoHEN, M. M. (1967). Continuous versus terminal visual feedback in prism aftereffects. Perceptual \& Motor Skills, 24, 1295-1302.

Cutting, J. E. (1986). Perception with an eye for motion. Cambridge, MA: MIT Press. 
Cutting, J. E. (1991). Four ways to reject directed perception. Ecological Psychology, 6, 185-204.

FitzPatrick, P., Carello, C., \& Turvey, M. T. (1994). Eigenvalues of the inertia tensor and exteroception by the "muscle sense." Neuroscience, 60,551-568.

GALlESE, V. (2003). A neuroscientific grasp of concepts: From control to representation. Philosophical Transactions of the Royal Society of London: Series B, 358, 1231-1240.

GIBSON, E. J., \& BERGMAN, R. (1954). The effect of training on absolute estimation of distance over the ground. Journal of Experimental Psychology, 48, 473-480.

GiBson, J. J. (1966). The senses considered as perceptual systems. Boston: Houghton Mifflin.

Gibson, J. J. (1979). The ecological approach to visual perception. Boston: Houghton Mifflin.

Hamilton, C. R. (1964). Intermanual transfer of adaptation to prisms. American Journal of Psychology, 77, 457-462.

HARRIS, C. S. (1963). Adaptation to displaced vision: Visual, motor, or proprioceptive change? Science, 140, 812-813.

HARRIS, C. S. (1965). Perceptual adaptation to inverted, reversed, and displaced vision. Psychological Review, 72, 419-444.

Heвв, D. O. (1949). The organization of behavior: A neuropsychological theory. New York: Wiley.

Heft, H. (1993). A methodological note on overestimates of reaching distance: Distinguishing between perceptual and analytical judgments. Ecological Psychology, 5, 255-271.

JACOBS, D. M., \& MichaELS, C. F. (2005). The education of attention and calibration in one-handed catching. Manuscript submitted for publication.

KINGMA, I., BEeK, P. J., \& VAN DieËN, J. H. (2002). The inertia tensor versus static moment and mass in perceiving length and heaviness of hand-wielded rods. Journal of Experimental Psychology: Human Perception \& Performance, 28, 180-191.

Kugler, P. N., Kelso, J. A. S., \& TuRveY, M. T. (1980). On the concept of coordinative structures as dissipative structures: I. Theoretical lines of convergence. In G. E. Stelmach \& J. Requin (Eds.), Tutorials in motor behavior (pp. 3-47). Amsterdam: North-Holland.

Kugler, P. N., \& Turvey, M. T. (1987). Information, natural law, and the self-assembly of rhythmic movement. Hillsdale, NJ: Erlbaum.

LASHLEY, K. S. (1930). Basic neural mechanisms in behavior. Psychological Review, 37, 1-24.

LURIA, A. R. (1973). The working brain. Harmondsworth: Penguin.

MARK, L. (1987). Eyeheight-scaled information about affordances: A study of sitting and stair climbing. Journal of Experimental Psychology: Human Perception \& Performance, 13, 361-370.

PaGano, C. C., FitzPatrick, P., \& Turvey, M. T. (1993). Tensorial basis to the constancy of perceived object extent over variations of dynamic touch. Perception \& Psychophysics, 54, 43-54.

Redding, G. M., \& Wallace, B. (1988). Components of prism adaptation in terminal and concurrent exposure: Organization of the eye-hand coordination loop. Perception \& Psychophysics, 44, 59-75.

Redding, G. M., \& Wallace, B. (1997). Adaptive spatial alignment. Mahwah, NJ: Erlbaum.

REED, E. S. (1982). An outline of a theory of action systems. Journal of Motor Behavior, 14, 98-134.

REED, E. S. (1996). Encountering the world: Toward an ecological psychology. New York: Oxford University Press.

Rieser, J. J., Pick, H. L., Ashmead, D. H., \& Garing, A. E. (1995). Calibration of human locomotion and models of perceptual-motor organization. Journal of Experimental Psychology: Human Perception \& Performance, 21, 480-497.

RiLEY, M. A., \& TURVEY, M. T. (2001). Inertial constraints on limb proprioception are independent of visual calibration. Journal of Experimental Psychology: Human Perception \& Performance, 27, 438-455.

Solomon, H. Y., \& TurveY, M. T. (1988). Haptically perceiving the distance reachable with hand-held objects. Journal of Experimental Psychology: Human Perception \& Performance, 14, 404-427.

Turvey, M. T. (1996). Dynamic touch. American Psychologist, $\mathbf{5 1}_{2}$ 1134-1152.

Turvey, M. T., \& Carello, C. (1995). Dynamic touch. In W. Epstein \& S. Rogers (Eds.), Handbook of perception and cognition: Perception of space and motion (pp. 401-490). New York: Academic Press.

Wagman, J. B., Shockley, K., Riley, M. A., \& Turvey, M. T. (2001). Attunement, calibration, and exploration in fast haptic perceptual learning. Journal of Motor Behavior, 33, 323-327.

Wallace, B., \& ReDding, G. M. (1979). Additivity in prism adaptation as manifested in intermanual and interocular transfer. Perception \& Psychophysics, 25, 133-136.

Withagen, R., \& MiChaELS, C. F. (2001, June). The calibration of haptically perceived length to visually perceived reachable distance. Poster presented at the Eleventh International Conference on Perception and Action, Storrs, CT.

Withagen, R., \& Michaels, C. F. (2002). The calibration of walking transfers to crawling: Are action systems calibrated? Ecological Psychology, 14, 223-234.

\section{NOTES}

1. Multiple realizability of function in perception has also been proposed at another level. The theory of directed perception (Cutting, $1986,1991)$ proposes that different information variables can constrain the same perception on different occasions. Our article does not address this level.

2. The common measures for calibration are constant error and variable error (see, e.g., E. J. Gibson \& Bergman, 1954; Wagman et al., 2001). The former refers to the mean percentage error; the latter describes the variability of the percepts. Note that both the slope and the intercept of the regression lines relate to the constant error. Hence, using slope and intercept instead of constant error as dependent measures in our analyses yields a more detailed picture of the calibration effects.

3. Obviously, the value of the information variables is identical in Experiments 1 and 2, so the "false" feedback specified that the rods were made of a lighter material than the $0.67 \mathrm{~g} / \mathrm{cm}^{3}$ material used in the rods of Experiment 1.

4. To test whether not including Participant 6 qualitatively affected our findings, we redid the ANOVAs on the slopes and intercepts. The ANOVA on the slopes again revealed an effect of condition $[F(1,8)=$ $53.12, p<.0001]$, no effect of hand $[F(1,8)=.005, p>.1]$, and no condition $\times$ hand interaction $[F(1,8)=.38, p>.1]$. The repeated measures ANOVA on the intercepts revealed no significant effects $(p>.5)$. Thus, even when Participant 6 is included, we can conclude that length perception by dynamic touch was recalibrated and transferred from the right hand to the left hand.

(Manuscript received January 17, 2003; revision accepted for publication January 5, 2004.) 